\title{
Multiple-Symbol Differential Sphere Detection Aided Differential Space-Time Block Codes Using QAM Constellations
}

\author{
Chao Xu, Student Member, IEEE, Li Wang, Soon Xin Ng, Senior Member, IEEE, and Lajos Hanzo, Fellow, IEEE
}

\begin{abstract}
We propose Multiple-Symbol Differential Detection (MSDD) aided Differential Space-Time Block Codes (DSTBCs) using QAM. Our solution eliminates the requirement of high-complexity MIMO channel estimation and its pilot overhead, while mitigating the potential performance degradation of noncoherent receivers in fast fading channels. Additionally, we develop Multiple-Symbol Differential Sphere Detection (MSDSD) for the QAM aided DSTBC schemes advocated, in order to circumvent their potentially excessive complexity.
\end{abstract}

Index Terms-Differential space-time block code, multiple-symbol differential sphere detection, QAM.

\section{INTRODUCTION}

$\mathbf{S}$ PACE-TIME BLOCK CODES (STBCs) [1], [2] provide an effective means of mitigating the deleterious effects of channel fading. However, most of the classic coherent MIMO detection literature stipulated the idealized assumption that perfect Channel State Information (CSI) is available at the receiver. In reality, as the number of links increases, obtaining accurate CSI imposes a dramatically increased channel estimation complexity, especially for high speed vehicles. Hence Differential STBCs (DSTBCs) using PSK were proposed in [3], [4], where the potentially high pilot overhead was eliminated. The most challenging task of DSTBC design is to construct unitary transmission matrices and as a result, the DSTBCs using nonconstant modulus constellations proposed in [5] required both power normalization at the transmitter as well as fading channel's power envelope estimation at the receiver.

In the absence of channel estimation, the Conventional Differential Detection (CDD) suffers from a $3 \mathrm{~dB}$ performance penalty. Furthermore, upon increasing the Doppler frequency, an irreducible error floor may be formed. To circumvent this problem, Multiple-Symbol Differential Detection (MSDD) designed for Differential PSK (DPSK) as well as for PSK-aided DSTBCs was proposed in [6], [7], where a sufficiently long detection window of $N_{w} \geq 2$ was employed in order to make a joint decision concerning $N_{w}$ consecutive symbols. However, the MSDD complexity increases exponentially as $N_{w}$ increases.

Manuscript received April 19, 2011; revised June 11, 2011; accepted June 19, 2011. Date of publication June 27, 2011; date of current version July 11, 2011. This work was supported by the RC-UK under the auspices of the India-UK Advanced Technology Centre (IU-ATC), the EPSRC under the China-UK science bridgeand by the the EU's Concerto project. The associate editor coordinating the review of this manuscript and approving it for publication was Dr. Yong Liang Guan.

The authors are with the School of Electronics and Computer Science, University of Southampton, Southampton, SO17 1BJ, U.K. (e-mail: (cx1g08@ecs. soton.ac.uk; 1w5@ecs.soton.ac.uk; sxn@ecs.soton.ac.uk; 1h@ecs.soton.ac.uk).

Digital Object Identifier 10.1109/LSP.2011.2160851
As a remedy, Multiple-Symbol Differential Sphere Detection (MSDSD) was introduced in [8], [9]. Given this background, the design of MSDD/MSDSD for QAM-aided DSTBCs is an open problem at the time of writing. A challenge is that nonconstant-modulus QAM results in a received signal power fluctuation, which has to be taken into account by the MSDD/MSDSD. Against this background, the novel contributions of this letter are as follows.

1) In Section II, we further develop the CDD aided DSTBCs of [5] using QAM. Our solution eliminates the need for fading channel power estimation at the receiver.

2) The MSDD designed for QAM-aided DSTBCs is proposed in Section III, where the channel correlation is updated according to the hypothesized combinations of the transmitted symbols.

3) We further develop the MSDSD for the family of DSTBCs using QAM in order to mitigate the MSDD complexity.

The following notations are used throughout the paper. We assume that $M=2$ transmit antenna elements and a variable number of $N$ receive antenna elements are employed. Furthermore, $N_{w}$ refers to the MSDD/MSDSD window duration.

\section{Conventional Differential Detection}

\section{A. Preliminaries}

The differential encoding process of DSTBCs using $L$-PSK/QAM is expressed as [5]

$$
\begin{aligned}
& \underbrace{\left[\begin{array}{cc}
s_{n}^{1} & s_{n}^{2} \\
-\left(s_{n}^{2}\right)^{*} & \left(s_{n}^{1}\right)
\end{array}\right]}_{\mathbf{S}_{n}}=\frac{1}{\theta_{n-1}} \underbrace{\left[\begin{array}{cc}
x_{n-1}^{1} & x_{n-1}^{2} \\
-\left(x_{n-1}^{2}\right)^{*} & \left(x_{n-1}^{1}\right)^{*}
\end{array}\right]}_{\mathbf{X}_{n-1}}
\end{aligned}
$$

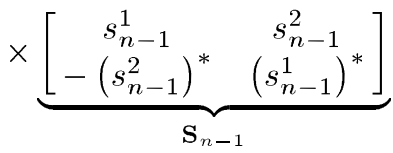

where $\left(x_{n-1}^{1}, x_{n-1}^{2}\right)$ are the modulated $L$-PSK/QAM symbols, which are scaled by a constant multipler of $1 / \sqrt{M}$. The power normalization factor of $\left(1 / \theta_{n-1}\right)$ in (1) is defined by $\theta_{n-1}=$ $\sqrt{\left\|\mathbf{S}_{n-1}\right\|^{2} / M}=\sqrt{\left|s_{n-1}^{1}\right|^{2}+\left|s_{n-1}^{2}\right|^{2}}$, which is equivalent to $\theta_{n-1}=\sqrt{\left\|\mathbf{X}_{n-2}\right\|^{2} / M}=\sqrt{\left|x_{n-2}^{1}\right|^{2}+\left|x_{n-2}^{2}\right|^{2}}$. This relationship ensures that the transmission matrix power would not keep on decreasing, when high-order QAM is employed.

The signal arriving at the receiver is modeled as

$$
\mathbf{Y}_{n}=\mathbf{S}_{n} \mathbf{H}_{n}+\mathbf{V}_{n}
$$

${ }^{1}$ We note that for constant-modulus constellations the factor $\theta_{n-1}$ remains a constant of 1.0 all the time. 
where the received signal matrix $\mathbf{Y}_{n}$ has $(M \times N)$ elements and the Rayleigh fading channel matrix $\mathbf{H}_{n}$ is generated according to Clarke's fading model [6]. The Additive White Gaussian Noise (AWGN) term $\mathbf{V}_{n}$ has a zero mean and a variance of $N_{0}$ in each dimension.

\section{B. CDD Aided DSTBCs Using QAM}

The CDD generally assumes that the fading channel remains constant for two DSTBC transmission durations, i.e., we have $\left(\mathbf{H}_{n} \approx \mathbf{H}_{n-1}\right)$. The detector designed for the DSTBCs using QAM [5] may be summarized as

$$
\begin{aligned}
\tilde{x}_{n-1}^{1} & =\mathbf{Y}_{n}^{1}\left(\mathbf{Y}_{n-1}^{1}\right)^{H}+\mathbf{Y}_{n-1}^{2}\left(\mathbf{Y}_{n}^{2}\right)^{H} \\
& =\left(\left\|\mathbf{H}_{n}^{1}\right\|^{2}+\left\|\mathbf{H}_{n}^{2}\right\|^{2}\right) \cdot \theta_{n-1} \cdot x_{n-1}^{1}+\tilde{V}_{n}^{1}, \\
\tilde{x}_{n-1}^{2} & =\mathbf{Y}_{n}^{1}\left(\mathbf{Y}_{n-1}^{2}\right)^{H}-\mathbf{Y}_{n-1}^{1}\left(\mathbf{Y}_{n}^{2}\right)^{H} \\
& =\left(\left\|\mathbf{H}_{n}^{1}\right\|^{2}+\left\|\mathbf{H}_{n}^{2}\right\|^{2}\right) \cdot \theta_{n-1} \cdot x_{n-1}^{2}+\tilde{V}_{n}^{2}
\end{aligned}
$$

where the $(1 \times N)$-element vectors $\left\{\mathbf{Y}_{n}^{m}\right\}_{m=1}^{M}$ and $\left\{\mathbf{H}_{n}^{m}\right\}_{m=1}^{M}$ denote the $m$ th row of $\mathbf{Y}_{n}$ and $\mathbf{H}_{n}$ in (2), while the equivalent noise terms $\tilde{V}_{n}^{1}$ and $\tilde{V}_{n}^{2}$ are given by

$$
\begin{aligned}
\tilde{V}_{n}^{1}= & {\left[\begin{array}{ll}
s_{n}^{1} & s_{n}^{2}
\end{array}\right]\left[\begin{array}{cc}
\mathbf{H}_{n}^{1} & \left(\mathbf{H}_{n}^{2}\right)^{*} \\
\mathbf{H}_{n}^{2} & -\left(\mathbf{H}_{n}^{1}\right)^{*}
\end{array}\right]\left[\begin{array}{c}
\left(\mathbf{V}_{n-1}^{1}\right)^{H} \\
\left(\mathbf{V}_{n-1}^{2}\right)^{T}
\end{array}\right] } \\
& +\left[\begin{array}{ll}
\mathbf{V}_{n}^{1} & \left(\mathbf{V}_{n}^{2}\right)^{*}
\end{array}\right]\left[\begin{array}{cc}
\left(\mathbf{H}_{n}^{1}\right)^{H} & \left(\mathbf{H}_{n}^{2}\right)^{H} \\
\left(\mathbf{H}_{n}^{2}\right)^{T} & -\left(\mathbf{H}_{n}^{1}\right)^{T}
\end{array}\right] \\
& \times\left[\begin{array}{cc}
\left(s_{n-1}^{1}\right)^{*} \\
\left(s_{n-1}^{2}\right)^{*}
\end{array}\right]+\left[\begin{array}{ll}
\mathbf{V}_{n}^{1} & \left(\mathbf{V}_{n}^{2}\right)^{*}
\end{array}\right]\left[\begin{array}{c}
\left(\mathbf{V}_{n-1}^{1}\right)^{H} \\
\left(\mathbf{V}_{n-1}^{2}\right)^{T}
\end{array}\right], \\
\tilde{V}_{n}^{2}= & {\left[\begin{array}{ll}
s_{n}^{1} & s_{n}^{2}
\end{array}\right]\left[\begin{array}{ll}
\mathbf{H}_{n}^{1} & \left(\mathbf{H}_{n}^{2}\right)^{*} \\
\mathbf{H}_{n}^{2} & -\left(\mathbf{H}_{n}^{1}\right)^{*}
\end{array}\right]\left[\begin{array}{c}
\left(\mathbf{V}_{n-1}^{2}\right)^{H} \\
-\left(\mathbf{V}_{n-1}^{1}\right)^{T}
\end{array}\right] } \\
& +\left[\begin{array}{ll}
\mathbf{V}_{n}^{1} & \left(\mathbf{V}_{n}^{2}\right)^{*}
\end{array}\right]\left[\begin{array}{cc}
\left(\mathbf{H}_{n}^{1}\right)^{H} & \left(\mathbf{H}_{n}^{2}\right)^{H} \\
\left(\mathbf{H}_{n}^{2}\right)^{T} & -\left(\mathbf{H}_{n}^{1}\right)^{T}
\end{array}\right] \\
& \times\left[\begin{array}{cc}
-s_{n-1}^{2} \\
s_{n-1}^{1}
\end{array}\right]+\left[\begin{array}{ll}
\mathbf{V}_{n}^{1} & \left(\mathbf{V}_{n}^{2}\right)^{*}
\end{array}\right]\left[\begin{array}{c}
\left(\mathbf{V}_{n-1}^{2}\right)^{H} \\
-\left(\mathbf{V}_{n-1}^{1}\right)^{T}
\end{array}\right]
\end{aligned}
$$

where the $(1 \times N)$-element vectors $\left\{\mathbf{V}_{n}^{m}\right\}_{m=1}^{2}$ refer to the $m$ th row of the AWGN matrix $\mathbf{V}_{n}$ in (2).

In order to recover the QAM symbols $\left(x_{n-1}^{1}, x_{n-1}^{2}\right)$ from $\left(\tilde{x}_{n-1}^{1}, \tilde{x}_{n-1}^{2}\right)$ of (3), both the normalization factor $\theta_{n-1}$ and the fading channel's power envelope of $p=\left\|\mathbf{H}_{n}^{1}\right\|^{2}+\left\|\mathbf{H}_{n}^{2}\right\|^{2}$ has to be known by the QAM demodulator. The factor $\theta_{n-1}$ may be obtained from the previous decisions, but the factor $p$ has to be estimated by evaluating the auto-correlation of the received signal [5]. Naturally, the accuracy of this estimation highly depends on both the estimation window duration as well as on the Doppler frequency.

However, the fading channel power estimation required by [5] can be avoided. Based on the usual CDD assumption of $\left(\mathbf{H}_{n} \approx \mathbf{H}_{n-1}\right)$, (2) may be further formulated as

$$
\begin{aligned}
\mathbf{Y}_{n} & =\frac{1}{\theta_{n-1}} \mathbf{X}_{n-1} \mathbf{S}_{n-1} \mathbf{H}_{n}+\mathbf{V}_{n} \\
& \approx \frac{1}{\theta_{n-1}} \mathbf{X}_{n-1}\left(\mathbf{Y}_{n-1}-\mathbf{V}_{n-1}\right)+\mathbf{V}_{n}
\end{aligned}
$$

which requires the extension of the CDD decision metric from the one introduced in [10]

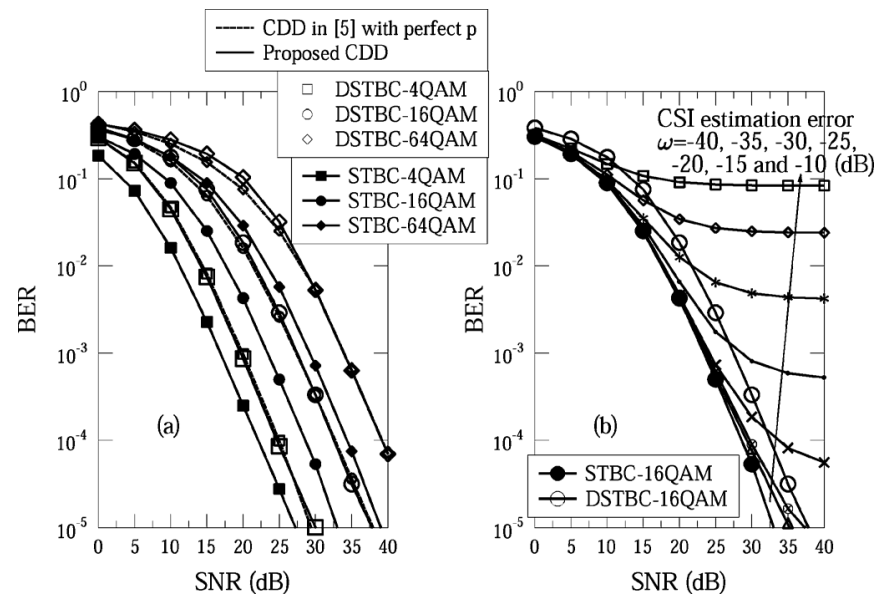

Fig. 1. BER performance of CDD aided DSTBCs using L-QAM in comparison to (a) the CDD proposed in [5] assuming perfect fading channel power $(p)$ estimation; (b) its coherent counterpart in the presence of CSI estimation error, for $f_{d}=0.001$.

$$
\begin{aligned}
\mathbf{X}_{n-1}= & \arg \min _{\mathbf{X}_{n-1}}\left\|\mathbf{Y}_{n}-\frac{1}{\theta_{n-1}} \mathbf{X}_{n-1} \mathbf{Y}_{n-1}\right\|^{2} \\
= & \arg \max _{\mathbf{X}_{n-1}} \frac{2}{\theta_{n-1}} \operatorname{Re}\left\{\operatorname{tr}\left(\mathbf{X}_{n-1} \mathbf{Y}_{n-1} \mathbf{Y}_{n}^{H}\right)\right\} \\
& -\frac{\theta_{n}^{2}}{\theta_{n-1}^{2}}\left\|\mathbf{Y}_{n}\right\|^{2}
\end{aligned}
$$

where the normalization factor $\theta_{n-1}$ was obtained from the previous decisions. Compared to the symbol-based CDD of (3), our proposed CDD of (6) requires a full search over the expanded space-time constellation. However, the estimation of the fading channel power required by the CDD of (3) also imposes an extra detection complexity [5], which is eliminated by our proposed CDD of (6).

\section{Simulation Results}

The BER performance of the CDD aided DSTBCs employing $L$-QAM is portrayed in Fig. 1(a). It can be seen that our proposed CDD exhibits a similar BER performance to that of the CDD of [5], where the latter relies on perfect fading channel power $(p)$ estimation. We note that the noise power of $\tilde{V}_{n}$ in the CDD model of (3) is $\left(2 N_{0}+N_{0}^{2}\right)$ for each dimension, while the power of the equivalent noise term of our proposed CDD model in (6) is given by $\left(1+\left(1 / \theta_{n-1}\right)\right) N_{0}$, which implies that a small performance difference exits between the two CDD arrangements at low SNRs, especially when higher-order QAM is employed, as evidenced by Fig. 1(a) ${ }^{2}$. However, it was demonstrated in [5] that its CDD's performance highly relies on the accuracy of the fading channel power $p$ estimation. By contrast, our solution eliminates this estimation.

The performance of the family of coherent STBCs characterized in Fig. 1(a) relies on perfect CSI estimation, which is not realistic in practice. Hence it was demonstrated in Fig. 1(b) that the performance advantage of the coherent STBC schemes was eroded by the effects of realistic CSI estimation errors, which was modeled by the additive Gaussian noise of $C N(0, \omega)$.

\footnotetext{
${ }^{2}$ Since the powers of the equivalent noise terms are not exactly $2 N_{0}$, the performance gaps between the DSTBCs and STBCs seen in Fig. 1(a) are actually slightly higher than $3 \mathrm{~dB}$.
} 
Therefore, in the absence of accurate CSI estimation, the proposed noncoherent arrangement may be preferred.

\section{Multiple-Symbol Differential Detection}

\section{A. MSDD Aided DSTBCS Using QAM}

In order to avoid the performance degradation of the CDD in rapidly fading channels, in this section we propose an MSDD designed for QAM-aided DSTBCs. The MSDD observes $N_{w}$ received signal blocks, which may be formulated as

$$
\overline{\mathbf{Y}}=\overline{\mathbf{S}} \cdot \overline{\mathbf{H}}+\overline{\mathbf{V}}
$$

where the $N_{w}$-block received signal matrix $\overline{\mathbf{Y}}=\left[\mathbf{Y}_{n}^{T}, \ldots, \mathbf{Y}_{n-N_{w}+1}^{T}\right]^{T}$ has $\left(M N_{w} \times N\right)$ elements, and both the equivalent fading matrix $\overline{\mathbf{H}}=\left[\mathbf{H}_{n}^{T}, \ldots, \mathbf{H}_{n-N_{w}+1}^{T}\right]_{T}^{T}$ as well as the AWGN matrix $\overline{\mathbf{V}}=\left[\mathbf{V}_{n}^{T}, \ldots, \mathbf{V}_{n-N_{w}+1}^{T}\right]^{T}$ have the same size. The equivalent $N_{w}$-block transmission matrix $\overline{\mathbf{S}}=\operatorname{diag}\left\{\left[\mathbf{S}_{n}, \ldots, \mathbf{S}_{n-N_{w}+1}\right]\right\}$ has $\left(M N_{w} \times M N_{w}\right)$ elements. The MSDD aims for maximizing the a posteriori probability of [6]

$$
\operatorname{Pr}(\overline{\mathbf{Y}} \mid \overline{\mathbf{S}}) \propto \exp \left(-\operatorname{Tr}\left\{\overline{\mathbf{Y}}^{H} \mathbf{R}_{\overline{\mathbf{Y}} \mathbf{Y}}^{-\mathbf{Y}} \overline{\mathbf{Y}}\right\}\right)
$$

where the autocorrelation matrix $\mathbf{R}_{\overline{\mathbf{Y Y}}}$ is given by

$$
\begin{aligned}
\mathbf{R}_{\overline{\mathbf{Y Y}}} & =\overline{\mathbf{S}} \mathbf{R}_{\overline{\mathbf{H H}}} \overline{\mathbf{S}}^{H}+\mathbf{R}_{\overline{\mathbf{V V}}} \\
& =\hat{\mathbf{S}} \mathbf{C} \hat{\mathbf{S}}^{H}
\end{aligned}
$$

where we have $\mathbf{C}=\left(\mathbf{R}_{\hat{\mathbf{H}} \hat{\mathbf{H}}}+\mathbf{R}_{\overline{\mathbf{V} \mathbf{V}}}\right)$, and the equivalent unitary transmission matrix is now formulated by $\hat{\mathbf{S}}=\operatorname{diag}\left\{\left[\left(1 / \theta_{N_{w}}\right) \mathbf{S}_{N_{w}}, \ldots,\left(1 / \theta_{1}\right) \mathbf{S}_{1}\right]\right\}$, which implies that for each MSDD window, the first equivalent unitary transmission matrix is set to $\hat{\mathbf{S}}_{N_{w}}=\mathbf{I}_{\mathbf{M}}$, while the following transmission matrices may be expressed as

$$
\hat{\mathbf{S}}_{i}= \begin{cases}\frac{1}{\theta_{N_{w}-i+1}} \mathbf{X}_{N_{w}-i} \hat{\mathbf{S}}_{i+1}, & 1 \leq i<N_{w} \\ \mathbf{I}_{\mathbf{M}}, & i=N_{w} .\end{cases}
$$

This arrangement is designed for the benefit of backwards-oriented search in MSDSD. Meanwhile, the equivalent channel matrix in (9) is given by $\hat{\mathbf{H}}=\left[\theta_{N_{w}} \mathbf{H}_{N_{w}}^{T}, \ldots, \theta_{1} \boldsymbol{H}_{1}^{T}\right]^{T}$. It can be seen that the employment of nonconstant modulus QAM constellations imposes a transmit-symbol-dependent real-time variation on the correlation matrix $\mathbf{R}_{\hat{\mathbf{H}} \hat{\mathbf{H}}}$ recorded at the output of the fading channel, which is given by

$$
\mathbf{R}_{\hat{\mathbf{H}} \hat{\mathbf{H}}}=\left[\begin{array}{ccc}
\theta_{N_{w}}^{2} \boldsymbol{\Gamma}_{0} & \ldots & \theta_{N_{w}} \theta_{1} \boldsymbol{\Gamma}_{N_{w}-1} \\
\vdots & \ddots & \vdots \\
\theta_{1} \theta_{N_{w}} \boldsymbol{\Gamma}_{N_{w}-1} & \ldots & \theta_{1}^{2} \boldsymbol{\Gamma}_{0}
\end{array}\right]
$$

where we have $\left\{\boldsymbol{\Gamma}_{i}=N J_{0}\left(2 \pi f_{d} i\right) \mathbf{I}_{\mathbf{M}}\right\}_{i=1}^{N_{w}}$ according to Clarke's fading model [6], with $J_{0}$ denoting the zero-order Bessel function of the first kind and $f_{d}$ being the normalized Doppler frequency. Furthermore, the AWGN's correlation matrix in (9) is given by $\mathbf{R}_{\overline{\mathbf{V V}}}=N N_{0} \mathbf{I}_{\mathbf{M} \mathbf{N}_{\mathbf{w}}}$. Therefore, based on the a posteriori probability of (8), the MSDD decision metric may be formulated as

$$
\begin{aligned}
\hat{\mathbf{S}} & =\arg \min _{\hat{\mathbf{S}}} \operatorname{Tr}\left\{\overline{\mathbf{Y}}^{H}\left(\hat{\mathbf{S}} \mathbf{C} \hat{\mathbf{S}}^{H}\right)^{-1} \overline{\mathbf{Y}}\right\} \\
& =\arg \min _{\hat{\mathbf{S}}}\left\|\overline{\mathbf{Y}}^{H} \hat{\mathbf{S}} \mathbf{L}\right\|^{2}
\end{aligned}
$$

where the lower triangular matrix $\mathbf{L}$ is generated from the decomposition of $\mathbf{C}^{-1}=\mathbf{L} \mathbf{L}^{H}$. For each possible combination of $\hat{\mathbf{S}}$, the correlation matrix $\mathbf{R}_{\hat{\mathbf{H}} \hat{\mathbf{H}}}$ of (11) has to be evaluated according to the tentative power normalization factors $\left\{\theta_{i}\right\}_{i=1}^{N_{w}}$. If we use the number of the constellation points visited by MSDD/ MSDSD per detected DSTBC information matrix as a simple but tangible measure of complexity, then the MSDD complexity may be estimated by 3

$$
N_{v}=\frac{\left(L^{M}\right)^{\left(N_{w}-1\right)}}{N_{w}-1}
$$

which implies that the complexity of MSDD increases exponentially, as the window duration $N_{w}$ increases.

\section{B. MSDSD Aided DSTBCs Using QAM}

In order to mitigate the MSDD complexity, we propose MSDSD aided DSTBCs for QAM. The MSDSD designed for PSK-aided DSTBCs in [9] aims for minimizing the Partial Euclidean Distance (PED) metric derived by reformulating the MSDD Euclidean Distance (ED) term of (12) as

$$
\begin{aligned}
d_{i}^{2} & =\sum_{t=i}^{N_{w}-1}\left\|\sum_{j=t}^{N_{w}} l_{j t} \hat{\mathbf{S}}_{j}^{H} \overline{\mathbf{Y}}_{j}\right\|^{2} \\
& =d_{i+1}^{2}+\left\|l_{i i} \hat{\mathbf{S}}_{i}^{H} \overline{\mathbf{Y}}_{i}+\sum_{j=i+1}^{N_{w}} l_{j i} \hat{\mathbf{S}}_{j}^{H} \overline{\mathbf{Y}}_{j}\right\|^{2}
\end{aligned}
$$

where $\left\{\left\{l_{j i}\right\}_{j=1}^{N_{w}}\right\}_{i=1}^{N_{w}}$ is taken from the $\left(N_{w} \times N_{w}\right)$-element lower triangular matrix $\mathbf{L}$, which was generated from the inversion of the single-link channel correlation matrix $\mathbf{C}$ with the aid of $\mathbf{C}^{-1}=\mathbf{L L}^{H}$. Hence the single-link's output fading correlation matrix $\mathbf{R}_{\mathbf{h h}}$ should be obtained by substituting $(M=N=$ 1) into (11), while the single-link's AWGN correlation matrix is given by $\mathbf{R}_{\mathbf{v v}}=N_{0} \mathbf{I}_{\mathbf{N}_{\mathbf{w}}}$.

For nonconstant modulus QAM constellations, the PED increment term of (14) may be further extended as

$$
\begin{aligned}
\Delta_{i}^{2} & =\left\|l_{i i} \hat{\mathbf{S}}_{i}^{H} \overline{\mathbf{Y}}_{i}+\sum_{j=i+1}^{N_{w}} l_{j i} \hat{\mathbf{S}}_{j}^{H} \overline{\mathbf{Y}}_{j}\right\|^{2} \\
& =\left\|\frac{l_{i i}}{\theta_{N_{w}-i+1}} \mathbf{X}_{N_{w}-i}^{H} \overline{\mathbf{Y}}_{i}+\hat{\mathbf{S}}_{i+1}\left(\sum_{j=i+1}^{N_{w}} l_{j i} \hat{\mathbf{S}}_{j}^{H} \overline{\mathbf{Y}}_{j}\right)\right\|^{2} .
\end{aligned}
$$

The backward-oriented search of MSDSD [8] starts from the index $\left(i=N_{w}-1\right)$, and then the index is decreased. The PED increment of (15) should be minimized over $\mathbf{X}_{N_{w}-i}$. When the MSDSD search arrives at a specific index $i$, the previous transmission matrices $\left\{\hat{\mathbf{S}}_{j}\right\}_{j=i+1}^{N_{w}-1}$ have already been obtained from the previous decisions and their transmit power normalization factors $\left\{\theta_{N_{w}-j}\right\}_{j=i}^{N_{w}-1}$ have also been updated. However, the complete $\left(N_{w} \times N_{w}\right)$-element fading correlation matrix $\mathbf{R}_{\mathbf{h h}}$ still cannot be formed. Instead, a $\left[\left(N_{w}-i+1\right) \times\left(N_{w}-i+1\right)\right]$-element partial fading correlation matrix may be evaluated according to

\footnotetext{
${ }^{3}$ The number of the decomposition calculations of $C^{-1}$ is equal to the number of the constellation points visited by MSDD/MSDSD.
} 


$$
\tilde{\mathbf{R}}_{\mathbf{h h}}=\left[\begin{array}{ccc}
\theta_{N_{w}-i+1}^{2} \Gamma_{0} & \ldots & \theta_{N_{w}-i+1} \theta_{1} \Gamma_{N_{w}-i} \\
\vdots & \ddots & \vdots \\
\theta_{1} \theta_{N_{w}-i+1} \Gamma_{N_{w}-i} & \cdots & \theta_{1}^{2} \Gamma_{0}
\end{array}\right]
$$

where the new factor $\theta_{N_{w}-i+1}$ is updated according to the hypothesized information matrix $\mathbf{X}_{N_{w}-i}$, while the partial AWGN correlation matrix is given by $\tilde{\mathbf{R}}_{\mathbf{v v}}=N_{0} \mathbf{I}_{\left(\mathbf{N}_{\mathbf{w}}-\mathbf{i}+\mathbf{1}\right)}$. Accordingly, the partial channel correlation matrix $\tilde{\mathbf{C}}$ can be updated to $\tilde{\mathbf{C}}=\tilde{\mathbf{R}}_{\mathbf{h h}}+\tilde{\mathbf{R}}_{\mathrm{vv}}$, and the temporary lower triangular matrix is obtained according to $\tilde{\mathbf{L}} \tilde{\mathbf{L}}^{H}=\tilde{\mathbf{C}}^{-1}$. As a result, the PED increment of (15) should be updated as

$$
\begin{aligned}
\Delta_{i}^{2}=\| \frac{\tilde{l}_{1,1}}{\theta_{N_{w}-i+1}} \mathbf{X}_{N_{w}-i}^{H} \overline{\mathbf{Y}}_{i} & +\hat{\mathbf{S}}_{i+1} \\
\times & \left(\sum_{j=i+1}^{N_{w}} \tilde{l}_{j-i+1,1} \hat{\mathbf{S}}_{j}^{H} \overline{\mathbf{Y}}_{j}\right) \|^{2}
\end{aligned}
$$

where the elements $l_{j, i}$ of the complete lower triangular matrix $\mathbf{L}$ and the elements $\tilde{l}_{j-i+1,1}$ of the temporary lower trianglar matrix $\tilde{\mathbf{L}}$ are always exactly the same.

The detailed MSDSD search algorithm may be found in [8]. The resultant MSDSD complexity is lower-bounded by [11]

$$
N_{v} \geq \frac{\left(L^{M}\right)\left(N_{w}-1\right)}{N_{w}-1}=\left(L^{M}\right)
$$

where the lower bound can only be achieved either if $N_{w}=2$ is employed, or at high SNRs for $N_{w}>2$. However, at low SNRs, the MSDSD complexity still increases exponentially as $N_{w}$ increases [9]. This is because the sphere detector can only reduce its search radius sooner at high SNRs [12].

\section{Simulation Results}

The BER performance of the MSDSD aided DSTBC employing 16QAM is portrayed in Fig. 2(a). It can be seen that the error floor of the CDD imposed by rapidly fading channels is successfully mitigated by the proposed MSDSD. We note that the MSDSD associated with $N_{w}=2$ is equivalent to the CDD. Furthermore, as the MSDSD window duration increases, the performance of the noncoherent receiver approaches that of the coherent scheme relying on perfect CSI estimation, as evidenced by Fig. 2(a).

Fig. 2(b) presents our complexity comparison between the MSDD and the MSDSD. As mentioned, the MSDD complexity of (13) increases exponentially with $N_{w}$, which makes the MSDD having a window length of $N_{w}>3$ complex. By contrast, the MSDSD exhibits a reduced complexity in Fig. 2(b), and the complexity lower bound of (18) is achieved by the MSDSD at high SNRs.

\section{CONCLUSION}

We further developed the CDD of [5] designed for DSTBCs using nonconstant modulus QAM constellations. Our solution eliminates the requirement of fading channel power estimation.
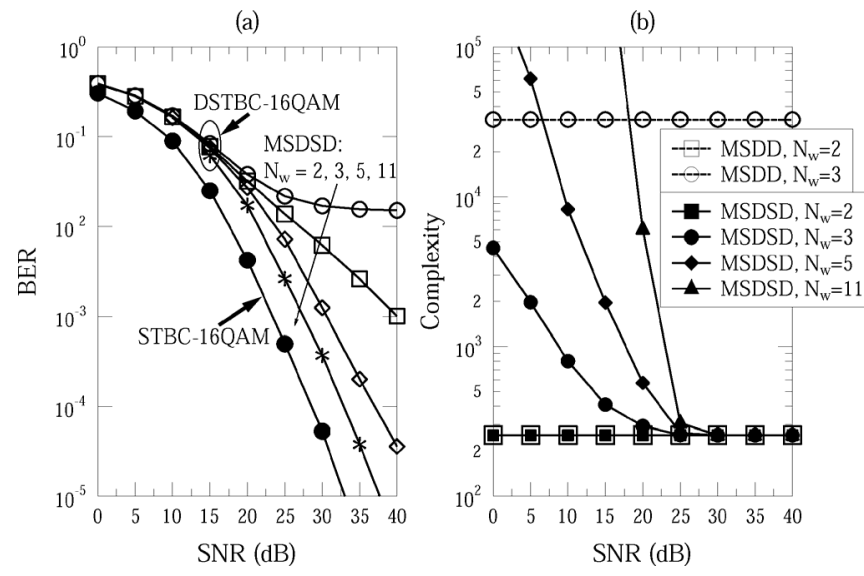

Fig. 2. BER performance and Complexity of MSDSD aided DSTBC employing 16QAM, for $f_{d}=0.03$.

Furthermore, we proposed the MSDD as well as the MSDSD designed for QAM aided DSTBCs. Our simulation results demonstrated that the error floor of noncoherent receivers encountered in rapidly fading channels was successfully mitigated by our MSDSD, which also exhibited a reduced complexity compared to the proposed MSDD arrangement.

\section{ACKNOWLEDGMENT}

The support of the RC-UK under the auspices of the India-UK Advanced Technology Centre (IU-ATC) and the EPSRC under the China-UK science bridge, as well as that of the EU's Concerto project, is gratefully acknowledged.

\section{REFERENCES}

[1] S. Alamouti, "A simple transmit diversity technique for wireless communications," IEEE J. Sel. Areas Commun., vol. 16, pp. 1451-1458, Oct. 1998.

[2] V. Tarokh, H. Jafarkhani, and A. Calderbank, "Space-time block codes from orthogonal designs," IEEE Trans. Inf. Theory, vol. 45, pp. 1467-1456, Jul. 1999.

[3] V. Tarokh and H. Jafarkhani, "A differential detection scheme for transmit diversity," IEEE J. Sel. Areas Commun., vol. 18, pp. 1169-1174, Jul. 2000

[4] G. Ganesan and P. Stoica, "Differential modulation using space-time block codes," IEEE Signal Process. Lett., vol. 9, pp. 57-60, Feb. 2002.

[5] C.-S. Hwang, S. H. Nam, J. Chung, and V. Tarokh, "Differential space time block codes using nonconstant modulus constellations," IEEE Trans. Signal Process., vol. 51, pp. 2955-2964, Nov. 2003.

[6] P. Ho and D. Fung, "Error performance of multiple-symbol differential detection of PSK signals transmitted over correlated Rayleigh fading channels," IEEE Trans. Commun., vol. 40, pp. 1566-1569, Oct. 1992.

[7] R. Schober and L.-J. Lampe, "Noncoherent receivers for differential space-time modulation," IEEE Trans. Commun., vol. 50, pp. 768-777, May 2002

[8] L. Lampe, R. Schober, V. Pauli, and C. Windpassinger, "Multiplesymbol differential sphere decoding," IEEE Trans. Commun., vol. 53, pp. 1981-1985, Dec. 2005.

[9] V. Pauli and L. Lampe, "On the complexity of sphere decoding for differential detection," IEEE Trans. Inf. Theory, vol. 53, pp. 1595-1603, Apr. 2007

[10] C. Yuen, Y. L. Guan, and T. T. Tjhung, "Unitary differential space-time modulation with joint modulation," IEEE Trans. Veh. Technol., vol. 56, pp. 3937-3944, Nov. 2007.

[11] L. Hanzo, Y. Akhtman, M. Jiang, and L. Wang, MIMO-OFDM for LTE, WIFI and WIMAX: Coherent Versus Non-Coherent and Cooperative Turbo-Transceivers. Hoboken, NJ: Wiley, 2010.

[12] D. Pham, K. Pattipati, P. Willett, and J. Luo, "An improved complex sphere decoder for V-BLAST systems," IEEE Signal Process. Lett., vol. 11, pp. 748-751, Sep. 2004. 